\title{
A comparison between laparoscopic and robotic- assisted laparoscopic pyeloplasty in patients with ureteropelvic junction obstruction
}

\section{Niwat Lukkanawong}

Department of Urology, Charoenkrung Pracharak Hospital

Masashi Honda ( $\sim$ honda@tottori-u.ac.jp)

Tottori University Faculty of Medicine https://orcid.org/0000-0002-0358-676X

Shogo Teraoka

Department of Urology, Tottori University Faculty of Medicine

\section{Yusuke Kimura}

Department of Urology, Tottori University Faculty of Medicine

\section{Tetsuya Yumioka}

Department of Urology, Tottori University Faculty of Medicine

Hideto Iwamoto

Department of Urology, Tottori University Faculty of Medicine

Shuichi Morizane

Department of Urology, Tottori University Faculty of Medicine

Katsuya Hikita

Department of Urology, Tottori University Faculty of Medicine

Atsushi Takenaka

Department of Urology, Tottori University Faculty of Medicine

\section{Research article}

Keywords: Pyeloplasty, Laparoscopic surgery, Robotic surgery

Posted Date: October 19th, 2020

DOI: https://doi.org/10.21203/rs.3.rs-79979/v1

License: (c) (i) This work is licensed under a Creative Commons Attribution 4.0 International License. Read Full License 


\section{Abstract \\ Background}

The aim of this study is to compare the results of laparoscopic pyeloplasty and robotic-assisted laparoscopic pyeloplasty in patients with ureteropelvic junction obstruction.

\section{Methods}

Between March 2008 and May 2019, the patients who underwent retroperitoneal laparoscopic or roboticassisted laparoscopic pyeloplasty in our institution were retrospectively reviewed.

\section{Results}

Thirteen patients underwent laparoscopically, and 12 patients underwent robotic surgery. The significant difference was found in median operative time between laparoscopic group (296 minutes) and robotic group (199 minutes) $(P=0.001)$. The median time for drain removal in laparoscopic group was longer than robotic group ( 3 vs 2 days, respectively, $P=0.029$ ).

\section{Conclusions}

Laparoscopic and robotic-assisted laparoscopic pyeloplasty is safe and excellent success rates in patients with ureteropelvic junction obstruction. However, our experience study suggested that robotic surgery improves a total operative time, decreases drain removal time and less intraoperative blood loss than laparoscopic approach.

\section{Background}

In 1891, Kuster described the first successful pyeloplasty which has been considered the gold-standard treatment for ureteropelvic junction obstruction (UPJO), with success rate exceeding $90 \%(90-100 \%)$ [1 3]. Later within these two to three decades, a procedure of minimally invasive surgery (MIS) has been developed and gradually famous and become an accepted treatment option with varieties procedures including endoscopic pyelotomy, laparoscopic pyeloplasty (LP) and robotic-assisted laparoscopic pyeloplasty (RALP).

Although LP and RALP present success rates at a level comparable to those of open surgery, these two procedures has provided the advantages of lower morbidity, less postoperative pain, faster recovery time and shorter hospital stays [4 - 7]. Schuessler et al. [8] were the first one to do laparoscopic pyeloplasty in 1993. Since then, the procedure have published its popularity. However, anastomosis of ureteropelvic procedure is still considered as a hard and challenged for LP. The procedure demands advanced 
laparoscopic skill with a present of steep learning curve. These are limitations for a novice surgeon with low laparoscopic surgery skill.

With high technology development of robotic-assisted surgery, limitations occurred in LP surgery procedure have been gradually demolished. The procedure becomes less complex operative task, especially, reconstructive procedures for anastomosis suturing. These circumstances contribute towards the ongoing goodwill of RALP. However, robotic system exhibits limitations on lacking of tactile feedback sensation, wasting of docking time for setting up an assisting surgical robot instruments and highly supportive disbursement in several countries [9].

Up to date, there's still no confirmation that RALP has significant impact on outcomes over LP. To explore this issue, this retrospective study aimed to compare outcomes between LP and RALP in patients with UPJO by our single center.

\section{Methods}

\section{Design and setting}

The study protocol and approval to conduct this study was obtained from the local institutional research and ethics Board. The medical records of all patients who underwent LP or RALP due to UPJO between March 2008 and May 2019 were retrospectively reviewed. All patients were confirmed diagnosis of UPJO by renal ultrasonography (US), intravenous pyelogram (IVP) (optional), computerized tomography (CT) scan and a diuretic renogram. The indications for surgery are renal pain, urinary tract infection (UTI), stone formation, decrease renal function (split renal function $<40 \%$ ), obstructive pattern on diuretic renogram, progressive hydronephrosis on serial US examination and progressive loss of renal function on serial radionuclide renograms. The LP has been previously performed in 2008 through 2012. Since 2013 when the da Vinci ${ }^{\circledR}$ Si robotic system (Intuitive Surgical, Sunnyvale, CA) arrived in our hospital, all patients has been undergoing RALP.

The data collected from the chart review included patient demographics, preoperative evaluation, perioperative data (including total operative time, robotic docking time, and robotic console time), and postoperative results. Operative time was defined from the time of skin incision to skin closure, including docking time. Perioperative and postoperative complications were classified based on Clavien and Dindo classification [10]. The degree of hydronephrosis was grade of 0 to 3 according to Ellenbogen's grading system [11].

The primary outcome of our study was the success rate of surgery. This was defined as relief of symptoms as well as improve obstruction on a diuretic renogram at 6 to 12 months after surgery. The secondary outcome included operative time, length of hospitalization ( $\mathrm{LOH})$, estimated blood loss (EBL) and complications.

\section{Surgical procedure}


The Anderson-Hynes dismembered technique was performed in all patients for both LP and RALP group. The LP was performed retroperitoneally, whereas the RALP was an intraperitoneal approach. All patients were positioned in the standard flank position.

\section{Retroperitoneal laparoscopic pyeloplasty}

Our technique for LP has been described in the previous study [12]. A camera port was made in the middle axillary line at the midpoint between the 12th rib and the iliac crest. A balloon dissector was used to create retroperitoneal space, a $12-\mathrm{mm}$ trocar was placed. One working port was placed $7 \mathrm{~cm}$ dorsal to the camera port and two ports were placed in the anterior axillary line at the level of the iliac crest and in the subcostal region. Following exposure of the psoas muscle and the Gerota's fascia, the ureter was identified and mobilized to the ureteropelvic junction (UPJ). The stay suture was placed passed to the renal pelvis and pulled out through the abdominal wall. The ureter was spatulated laterally and the stenotic segment of the UPJ was resected. A $6 \mathrm{~F}$ ureteral stent was inserted into the ureter by antegrade fashion. The anastomosis was performed with a running suture using a 4-0 polydioxanone suture. The anterior anastomosis was initially completed, followed by the posterior anastomosis with the same suture. The crossing vessels were preserved by anterior transposition of ureter and pyelo-reduction was performed if necessary. If renal calculi were present, the stones were removed before anastomosis by laparoscopic graspers or by using flexible nephroscope with a stone basket through the 12-mm port.

\section{Robotic-assisted laparoscopic pyeloplasty}

The transperitoneal approach was our preferred in RALP. The position of the ports as shown in Fig. 1. A 12-mm camera port was made $6 \mathrm{~cm}$ lateral to the umbilicus by open Hasson technique. The 8-mm robotic arm ports were placed laterally at a distance of $8 \mathrm{~cm}$ from the camera port. The first assistant port was set at a distance of $6 \mathrm{~cm}$ from the midpoint between the caudal robot's arm port and the camera port. The da Vinci ${ }^{\circledR}$ robotic system was placed on the dorsal side of the patient.

The operation started with colon mobilization, allowing the colon to fall medially. Gerota's fascia was exposed. The ureter was identified and dissected up to UPJ. The rest of the surgical steps were almost the same as LP, except RALP did not need to stabilize the renal pelvis by stay sutures. Because of freedom of robotic arms, it was easy to perform anastomosis without any fixation.

\section{Follow up}

The ureteral stent was removed at 4 weeks after surgery. Follow up was scheduled for evaluation of clinical, urine examination and the renal US. A diuretic renogram was also performed at 6 to 12 months postoperatively.

\section{Statistical analysis}

We used the Student T-test for comparison between two groups that showed equal variance. On the other hand, the Mann-Whitney test was used between two groups showing non-normal distribution or non- 
uniform variance. In addition, in comparison of the ratio between the two groups, a chi-square test was used. For each test result, a corresponding two-sided $p$-value of $<0.05$ was considered statistically significant. All analyses were performed using SPSS software (IBM, Statistical Package for the Social Sciences ver 23, Chicago, IL USA).

\section{Results}

A total of 25 patients were included in this study. Thirteen and 12 patients underwent LP and RALP, respectively. Patient demographics and characteristics data are shown in Table 1. There were significantly higher BMI in LP group than RALP group $(P=0.012)$. However, the gender, age, side, clinical presentation, and history of previous surgery were similar in both groups. The crossing vessels were encountered in $5(39 \%)$ patients in LP group and 4 (33\%) patients in RALP group and they could be preserved in all cases. Two (15\%) patients in LP group had concomitant stones compared with $2(17 \%)$ patients in RALP group.

Table 1

Baseline demographics and disease characteristics $(n=25)$

\begin{tabular}{|c|c|c|c|}
\hline & LP, & RALP, & $P$-value \\
\hline & $n=13$ & $n=12$ & \\
\hline Age, yrs (range) ${ }^{\dagger}$ & $23(12-62)$ & $29(11-70)$ & 0.557 \\
\hline Sex: male/female & $7 / 6$ & $8 / 4$ & 0.688 \\
\hline $\mathrm{BMI}, \mathrm{kg} / \mathrm{m}^{2}$ (range) $^{\dagger}$ & $22.2(16.6-25.0)$ & $18.4(12.9-21.9)$ & 0.012 \\
\hline Side: right/left & $4 / 9$ & $4 / 8$ & 0.891 \\
\hline Presentations & & & 0.961 \\
\hline Pain & 9 & 9 & \\
\hline UTI & 2 & 1 & \\
\hline Hematuria & 1 & 1 & \\
\hline Incidental finding & 1 & 1 & \\
\hline Crossing vessels, $n(\%)$ & $5(38.5 \%)$ & $4(33.3 \%)$ & 0.79 \\
\hline Concomitant stones, $n(\%)$ & $2(15.4 \%)$ & $2(16.7 \%)$ & 0.93 \\
\hline Previous surgery, $n(\%)$ & $2(15.4 \%)$ & $0(0 \%)$ & 0.157 \\
\hline †: Age and BMI were expres & edian". & & \\
\hline $\begin{array}{l}\mathrm{BMI}=\text { body mass index; } \mathrm{LP} \\
\text { urinary tract infection }\end{array}$ & pic pyeloplasty; & obotic-assisted py & $\mathrm{y} ; \mathrm{UTI}=$ \\
\hline
\end{tabular}


The median operative time for RALP, including docking time, was significantly shorter than LP (296 minutes vs. 199 minutes in LP and RALP, respectively) $(P=0.001)$, while the median robotic docking time was 6 minutes (range 2 to 17) (Table 2). There was no significant difference in the median EBL between LP and RALP ( $5 \mathrm{ml}$ vs $0 \mathrm{ml}$, respectively) $(P=0.168)$. However, 2 cases in LP group had significant blood loss $(305$, and $600 \mathrm{ml})$, but all of them did not require a blood transfusion. Regarding complications, no intraoperative complications were observed in both groups. While in postoperative period, one patient in the RALP group was Clavien-Dindo level Illa. This patient had ureteral stent obstruction by a blood clot, which required to change stent by cystoscopy under local anesthesia. The median time for drain removal in LP group was 3 days (range 2 to 4), which is significantly more than 2 days (range 1 to 4 ) in RALP group $(P=0.029)$. The median length of hospital stay after surgery was similar in both groups were equivalent at 8 days $(P=0.677)$. The success rate, defined as resolution of symptoms and improvement of obstruction on a diuretic renogram, were similar at $100 \%$ for both groups. No open conversions were required for all patients. The median follow-up was 24 months.

Table 2

Perioperative and postoperative outcome

\begin{tabular}{|c|c|c|c|}
\hline & $\begin{array}{l}\text { LP, } \\
n=13\end{array}$ & $\begin{array}{l}\text { RALP, } \\
n=12\end{array}$ & $P$-value \\
\hline 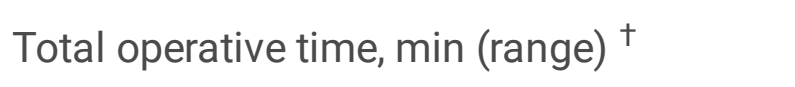 & $296(185-498)$ & $199(173-296)$ & 0.001 \\
\hline Robot docking time, min (range) ${ }^{\dagger}$ & & $6(2-17)$ & \\
\hline $\mathrm{EBL}, \mathrm{ml}$ (range) ${ }^{\dagger}$ & $5(0-600)$ & $0(0-50)$ & 0.168 \\
\hline \multicolumn{4}{|l|}{ Complications } \\
\hline Intraoperative & 0 & 0 & \\
\hline Postoperative: Clavien-Dindo Grade IIla, $n$ & 0 & 1 & \\
\hline Drain duration, days (range) ${ }^{+}$ & $3(2-4)$ & $2(1-4)$ & 0.029 \\
\hline Hospital stay, days (range) $^{\dagger}$ & $8(5-15)$ & $8(6-13)$ & 0.677 \\
\hline Conversion to open surgery, $n(\%)$ & 0 & 0 & \\
\hline Success rate, $n(\%)$ & $13(100 \%)$ & $12(100 \%)$ & 1 \\
\hline \multicolumn{4}{|c|}{$\begin{array}{l}\text { †: Total operative time, Robot docking time, EBL, Drain duration, and Hospital stay were expressed as } \\
\text { "Median". }\end{array}$} \\
\hline \multicolumn{4}{|c|}{ EBL = estimate blood loss; LP = laparoscopic pyeloplasty RALP = Robotic-assisted pyeloplasty } \\
\hline
\end{tabular}

\section{Discussion}


At the present, LP and RALP surgery have become alternative procedures with a minimally invasive surgery for the treatment of UPJO. The highly popular procedures displayed treatment effectiveness at a high level comparative to that of open pyeloplasty which once was the gold-standard surgery in the past [13]. Furthermore, these procedures provided the advantages of lower morbidity, less postoperative pain, and shorter hospital stays. Nevertheless, it was found that LP demanded high intracorporeal suturing skill and longer operative time comparative to open surgery [14].

After the robotic system has been introduced to operative treatment, various limitations on laparoscopic surgery could be demolished including decreased learning curve, reduced fatigue of a surgeon during surgery operation and increased effectiveness of operative treatment. With 3 dimensional visualization (3D) including freedom movement of robotic wrist, stable view, tremor filtering, and motion scaling, RALP procedure has gained higher popularity among surgeons $[15,16]$.

Braga has conducted a meta-analysis to compare LP vs. RALP treatments from 8 research studies which showed success rate exceeding $90 \%$, and 5 of which exhibited success rate at $100 \%$ for both LP and RALP. It was found that success rates of RALP were higher than those of LP ( $100 \%$ versus $97 \%$ and $99 \%$ versus $97 \%$ ) in 2 studies. In contrast, there was only one study reported that success rate of LP was greater than that of RALP (100\% versus $97 \%)$. However, the difference showed no statistical significance [17]. Moreover, our study found that success rates of both LP and RALP were equivalent at $100 \%$ when evaluation was performed from relief of symptoms and improve obstruction on a diuretic renogram. Furthermore, there was no open conversion discovered in both groups. Additionally, our study has disclosed that median operative time of LP was 296 (185-498) minutes, whilst that of RALP was 199 (173-296) minutes. The results suggested that RALP employed shorter total operative time comparative to that of LP with statistical difference. Light et al. [18] has performed a meta-analysis study to compare the operative time of LP and RALP in both children and adults from 14 research studies. The results indicated that there were 4 studies showing comparatively identical operative time in both LP and RALP. Whilst, there were 8 studies exhibiting shorter operative time in RALP compared to that of LP with statistical significance. In contrast, there were 3 studies showing that LP statistically significantly display shorter operative time comparative to RALP. According to the results from meta-analysis studies performed by Light et al., it can be concluded that RALP employed operative time shorter than LP for 27 minutes. Interestingly, it was believed that assisted robotic surgery involved shorter anastomosis suturing comparative to that of laparoscopic surgery [18].

However, Link et al. [19] has reported that LP displayed operative time shorter than that of RALP with statistical significance. Furthermore, RALP surgery could expedite anastomosis suturing procedure, though, some operative steps, such as colon reflection, consumed more time comparative to laparoscopic surgery since the robotic arms have been designed to operate with more effectiveness for precise than gross movements. Additionally, the robotic system consumed more time for robot docking and undocking [20]. Moreover, especial novice surgery center with low experience in robot-assisted surgery would consume more time for setting up procedure. Therefore, the operative time not only depended upon surgeons, but also experience and masterfulness of the entire robotic team [18, 21]. 
Lucas et al. [22] has reported a study comparing the surgery results by LP and RALP, performed in 274 and 465 patients, respectively. No statistically significant difference has been found in intraoperative complications, postoperative complications and anastomotic leak. In our study, intraoperative complications were not observed in both groups. However, it has been found that there was intraoperative significant blood loss among 2 patients operated by LP (305 and $600 \mathrm{ml}$, respectively), but blood transfusion was not required. Furthermore, one case in this group was previous performed balloon endopyelotomy with no success, therefore, it was difficult during LP surgery as a result of adhesion leading to renal parenchymal tear. Moreover, bleeding from inadequate renorrhaphy was indicated in another case in this group. Fortunately, laparoscopic suturing was achievably conducted to stop bleeding in both cases. For RALP group, there was 1 case discovered with postoperative complication from ureteral stent obstruction by blood clot. The patient was performed cystoscopy with stent changing under local anesthesia. Subsequently, there was no further postoperative complication found later.

In our research, we found that drain removal could be performed earlier in RALP group comparative to LP group with statistical significance. Median time for drain removal in RALP and LP groups were 3 (2-4) days and $2(1-4)$ days, respectively. Since the RALP surgery encouraged a surgeon to certainly qualify anastomosis suturing procedure comparative to that of LP, the drain removal in RALP group could be conducted earlier. The results from meta-analysis performed by Light et al. [18] has found that LOH in RALP was shorter than that in LP for 1.2 days. In contrast, our research discovered that it was comparatively the same between both groups, 8 (5-15) days for LP versus 8 (6-13) days for RALP. The causes of longer LOH in our research was that the patients were regularly allowed to stay in hospital until drain and Foley catheter were off and patients get normal routine activity. Some patients were worried of the distance between the hospital and their residences and demanded to stay longer at the hospital to assure their recovery. In comparison with a study of Patel et al. [23], which has reported 1.1 days for LOH, whilst the recovery time until the patients could continue all routine independent activities of daily living was 7.7 days, this recovery time and our LOH were comparatively similar to each other.

Limitations found in our research were defined. Since the study was performed with limited number of patients and the design of this study was retrospective review, then randomized control trial study was necessarily performed with more patients to confirm the results.

\section{Conclusions}

In era of minimally invasive surgery, LP and RALP treatments of UPJO have played critical roles and emerged widespread with equivalently qualified success rates comparatively the same to that of the standard open technique. The robotic surgical systems have been continuously developed to overcome the limitations of laparoscopic approach. In our study, we have clearly indicated that RALP displayed higher advantages than LP in the features of shorter operative time, less blood loss and earlier drain removal. These merits assure us of using RALP as excellent, effective and harmless alternative treatment.

\section{Abbreviations}


CT: Computerized tomography; EBL: Estimated blood loss; IVP: Intravenous pyelogram;

LOH: Length of hospitalization; LP: Laparoscopic pyeloplasty;

MIS: Minimally invasive surgery; RALP: Robotic-assisted laparoscopic pyeloplasty;

3D: 3 dimensional visualization; UPJ: Ureteropelvic junction;

UPJO: Ureteropelvic junction obstruction; US: Ultrasonography; UTI: Urinary tract infection

\section{Declarations}

\section{Acknowledgements}

The authors would like to thank Tottori University Hospital for providing the opportunity to conduct this study.

\section{Authors' contributions}

$\mathrm{NL}, \mathrm{MH}$, and ST contributed to the conception, study design, analysis, interpretation of data, and drafted the first manuscript. YK, TY, and $\mathrm{HI}$ contributed to acquisition of data. SM, KH, and AT supervised the study, helped to draft the manuscript, and were involved in revising it critically for important intellectual content. All authors read and approved the final manuscript.

\section{Funding}

None.

\section{Ethics approval and consent to participate}

The present study was approved by the Ethical Committee of Tottori University Faculty of Medicine. This was a retrospective study, and additional informed consent was not required by the board.

\section{Consent for publication}

Not Applicable.

\section{Competing interests}

The authors declare that they have no competing interests.

\section{References}

1. Eden CG. Minimally invasive treatment of ureteropelvic junction obstruction: a critical analysis of results. Eur Urol. 2007;52:983-989. 
2. Notley RG, Beaugie JM. The long-term follow-up of Anderson- Hynes pyeloplasty for hydronephrosis. Br J Urol. 1973;45:464-467.

3. Persky L, Krause JR, Boltuch RL. Initial complications and late results in dismembered pyeloplasty. J Urol. 1977;118:162-165.

4. Gill IS, Clayman RV, McDougall EM. Advances in urological laparoscopy. J Urol. 1995; 154:1275-1294.

5. Rassweiler J, Frede T, Henkel TO, Stock C, Alken P. Nephrectomy: a comparative study between the transperitoneal and retroperitoneal laparoscopic versus the open approach. Eur Urol. 1988;33:489-496.

6. Miyake H, Kawabata G, Gotoh A, Fujisawa M, Okada H, Arakawa S, et al. Comparison of surgical stress between laparoscopy and open surgery in the field of urology by measurement of humoral mediators. Int J Urol. 2002;9:329-333.

7. Simforoosh N, Basiri A, Tabibi A, Danesh AK, Sharifi-Aghdas F, Ziaee AM, et al. A comparison between laparoscopic and open pyeloplasty in patients with ureteropelvic junction obstruction. Urol J. 2004;1:165-169.

8. Schuessler WW, Grune MT, Tecuanhuey LV, Preminger GM. Laparoscopic dismembered pyeloplasty. J Urol. 1993;150:1795-1799.

9. Gupta NP, Nayyar R, Hemal AK, Mukherjee S, Kumar R, Dogra PN. Outcome analysis of robotic pyeloplasty: a large single-centre experience. BJU Int. 2010;105:980-983.

10. Clavien PA, Barkun J, de Oliveira ML, Vauthey JN, Dindo D, Schulick RD, et al. The Clavien-Dindo classification of surgical complications: five-year experience. Ann Surg. 2009;250:187-196.

11. Ellenbogen PH, Scheible FW, Talner LB, Leopold GR. Sensitivity of gray scale ultrasound in detecting urinary tract obstruction. Am J Roentgenol. 1978;130:731-733.

12. Isoyama T, Iwamoto H, Inoue S, Morizane S, Hinata N, Yao A, et al. Hydronephrosis after retroperitoneal laparoscopic dismembered Anderson-Hynes pyeloplasty in adult patients with ureteropelvic junction obstruction: A longitudinal analysis. Cent European J Urol. 2014;67:101-105.

13. Autorino R, Eden C, El-Ghoneimi A, Guazzoni G, Buffi N, Peters CA, et al. Robot-assisted and laparoscopic repair of ureteropelvic junction obstruction: a systematic review and meta-analysis. Eur Urol. 2014;65:430-452.

14. Jarrett TW, Chan DY, Charambura TC, Fugita O, Kavoussi LR. Laparoscopic pyeloplasty: the first 100 cases. J Urol. 2002;167:1253-1256.

15. Tasian GE, Casale P. The robotic-assisted laparoscopic pyeloplasty: gateway to advanced reconstruction. Urol Clin North Am. 2015;42:89-97.

16. Boysen WR, Gundeti MS. Robot-assisted laparoscopic pyeloplasty in the pediatric population: a review of technique, outcomes, complications, and special considerations in infants. Pediatr Surg Int. 2017;33:925-935. 
17. Braga LH, Pace K, DeMaria J, Lorenzo AJ. Systematic review and meta-analysis of robotic-assisted versus conventional laparoscopic pyeloplasty for patients with ureteropelvic junction obstruction: effect on operative time, length of hospital stay, postoperative complications, and success rate. Eur Urol. 2009;56:848-857.

18. Light A, Karthikeyan S, Maruthan S, Elhage O, Danuser H, Dasgupta P. Peri-operative outcomes and complications after laparoscopic vs robot-assisted dismembered pyeloplasty: a systematic review and meta-analysis. BJU Int. 2018;122:181-194.

19. Link RE, Bhayani SB, Kavoussi LR. A prospective comparison of robotic and laparoscopic pyeloplasty. Ann Surg. 2006;243:486-491.

20. Gettman MT, Neururer R, Bartsch G, Peschel R. Anderson-Hynes dismembered pyeloplasty performed using the da Vinci robotic system. Urology. 2002;60:509-513.

21. Esposito C, Masieri L, Castagnetti M, Sforza S, Farina A, Cerulo M, et al. Robot-assisted vs laparoscopic pyeloplasty in children with ureteropelvic junction obstruction (UPJO): technical considerations and results. J Pediatr Urol. 2019;15:667.e1-667.e8.

22. Lucas SM, Sundaram CP, Wolf JS, Leveillee JRJ, Bird VG, Aziz M, et al. Factors that impact the outcome of minimally invasive pyeloplasty: results of the Multi-Institutional Laparoscopic and Robotic Pyeloplasty Collaborative Group. J Urol. 2012;187:522-527.

23. Patel V. Robot-assisted laparoscopic dismembered pyeloplasty. Urology. 2005;66: 45-49.

\section{Figures}




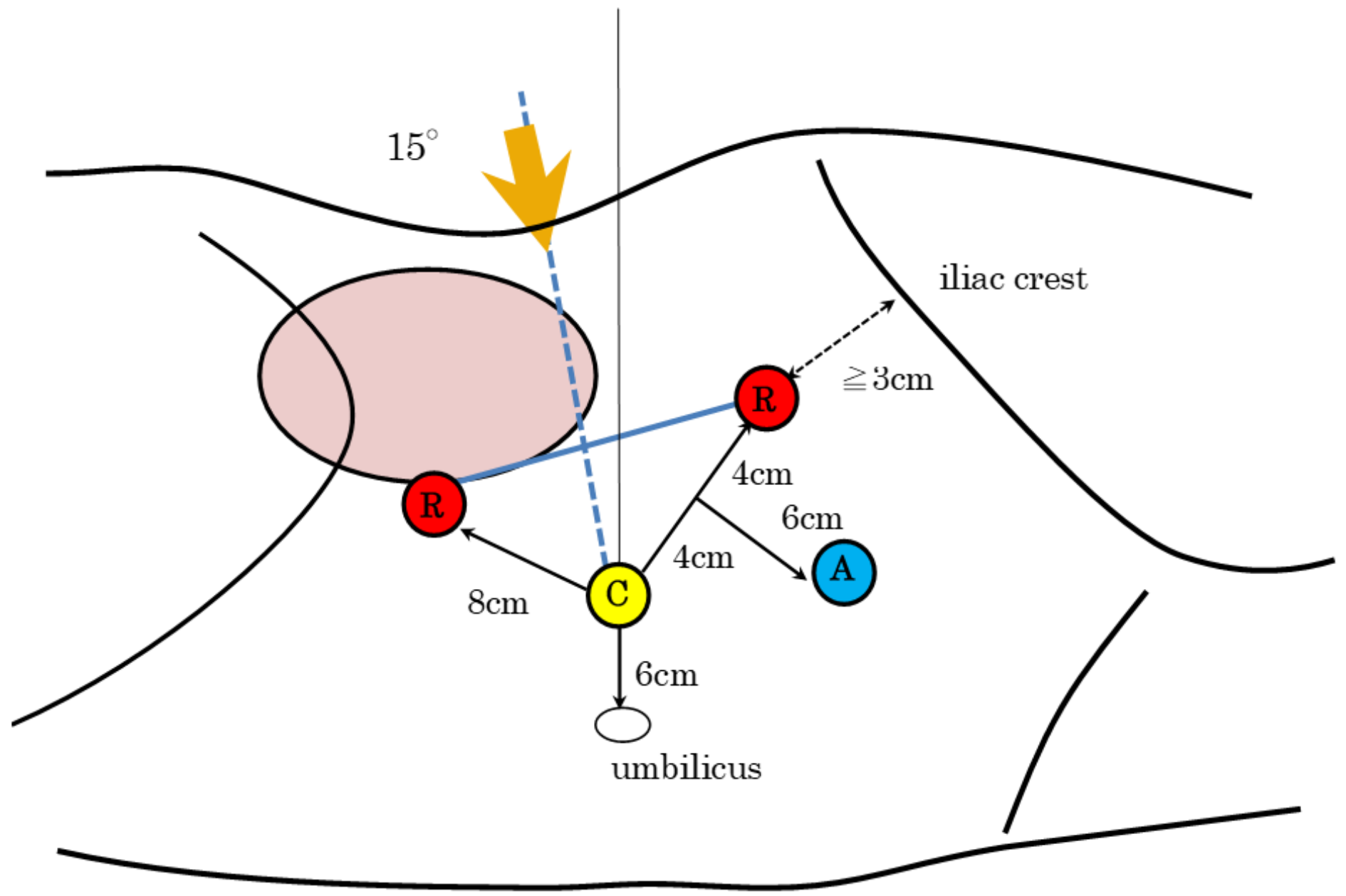

Figure 1

Trocar placement for left robotic-assisted pyeloplasty. $A=$ Assistant port $(12 \mathrm{~mm}) ; \mathrm{C}=$ Camera port (12 $\mathrm{mm}) ; \mathrm{R}=$ Robotic arm port $(8 \mathrm{~mm})$. 